\title{
Normal Cone Function Requires the Interphotoreceptor Retinoid Binding Protein
}

\author{
Ryan 0. Parker, ${ }^{1}$ Jie Fan, ${ }^{2}$ John M. Nickerson, ${ }^{3}$ Gregory I. Liou, ${ }^{4}$ and Rosalie K. Crouch ${ }^{2}$ \\ Departments of ${ }^{1}$ Neurosciences and ${ }^{2}$ Ophthalmology, Medical University of South Carolina, Charleston, South Carolina 29403, ${ }^{3}$ Department of \\ Ophthalmology, Emory University, Atlanta, Georgia 30322, and ${ }^{4}$ Department of Ophthalmology, Medical College of Georgia, Augusta, Georgia 30912
}

11-cis-retinal is the light-sensitive component in rod and cone photoreceptors, and its isomerization to all-trans retinal in the presence of light initiates the visual response. For photoreceptors to function normally, all-trans retinal must be converted back into 11-cis-retinal through a series of enzymatic steps known as the visual cycle. The interphotoreceptor retinoid-binding protein (IRBP) is a proposed retinoid transporter in the visual cycle, but rods in Irbp ${ }^{-1-}$ mice have a normal visual cycle. While rods are primarily responsible for dim light vision, the ability of cones to function in constant light is essential to human vision and may be facilitated by cone-specific visual cycle pathways. We analyzed the cones in Irbp ${ }^{-1-}$ mice to determine whether IRBP has a cone-specific visual cycle function. Cone electroretinogram (ERG) responses were reduced in $I r b p^{-1-}$ mice, but similar responses from $I r b p^{-1-}$ mice at all ages suggest that degeneration does not underlie cone dysfunction. Furthermore, cone densities and opsin levels in Irbp ${ }^{-1-}$ mice were similar to C57BL/6 (wild-type) mice, and both cone opsins were properly localized to the cone outer segments. To test for retinoid deficiency in Irbp ${ }^{-1-}$ mice, ERGs were analyzed before and after intraperitoneal injections of 9-cis-retinal. Treatment with 9-cis-retinal produced a significant recovery of the cone response in $I r b p^{-1-}$ mice and shows that retinoid deficiency underlies cone dysfunction. These data indicate that IRBP is essential to normal cone function and demonstrate that differences exist in the visual cycle of rods and cones.

\section{Introduction}

Photoreceptors utilize the photosensitive properties of 11-cis retinal to detect light. The 11-cis retinal in photoreceptors covalently binds a G-protein-coupled opsin to form the visual pigment molecules in rods and cones. In the dark, 11-cis retinal functions as an opsin inverse agonist and reduces opsin activity, but when light strikes a visual pigment, 11-cis retinal is isomerized to all-trans retinal, an opsin agonist (Wald, 1935, 1955). The photoisomerization of retinal allows photoreceptors to generate a cellular response to light, but for photoreceptors to function in constant light, new 11-cis retinal must be available to bind the opsin when the all-trans photoproduct is released. To meet this requirement, all-trans retinal is efficiently converted back to 11-cis retinal through the enzymatic steps of the visual cycle. Our understanding of the visual cycle is largely derived from studies of rods that mediate vision in dim light. Cones, which function in bright light and mediate the bulk of human vision, appear to utilize the classical visual cycle also but may have access to unique sources of

\footnotetext{
Received Jan. 6, 2009; revised Feb. 5, 2009; accepted March 7, 2009.

This study was supported by National Institutes of Health Grants EY04939 (R.K.C.), EY14793 [Medical University of South Carolina (MUSC) Vision Core], and EY016470, EY017045, and EY006360 (J.M.N.); Foundation Fighting Blindness, Inc. (Owings Mills, MD) (J.M.N., R.K.C.); and unrestricted awards to the Departments of Ophthalmology at MUSC and Emory University from Research to Prevent Blindness (RPB; New York). R.K.C. is an RPB Senior Scientific Investigator. R.0.P. is the recipient of an RPB Medical Student Eye Research Fellowship. We thank Patrice Goletz for technical assistance, Dr. Baerbel Rohrer for advice on ERGs, Dr. Luanna Bartholomew for editorial assistance, and Dr. Sharon Yeatts for statistical guidance.

Correspondence should be addressed to Ryan 0. Parker, Storm Eye Institute, Medical University of South Carolina, 167 Ashley Avenue, Charleston, SC 29403-5836. E-mail: parkerry@musc.edu.

D0I:10.1523/JNEUROSCI.0063-09.2009

Copyright $\odot 2009$ Society for Neuroscience $\quad$ 0270-6474/09/294616-06\$15.00/0
}

11-cis retinal that facilitate their function in constant light (Mata et al., 2002, 2005).

The classical visual cycle is a compartmentalized cascade with steps occurring in the photoreceptors and retinal pigment epithelium (RPE). Through its steps, all-trans photoproducts from the photoreceptors are passed to the RPE, converted back to 11-cisretinal, and returned to the photoreceptor for visual pigment regeneration. The compartmentalization of the visual cycle requires that poorly soluble and potentially toxic retinoids traverse the aqueous subretinal space between the photoreceptors and the $\mathrm{RPE}$, and interphotoreceptor retinoid-binding protein (IRBP) is thought to facilitate this process (Bunt-Milam and Saari, 1983; Fong et al., 1984; Pepperberg et al., 1993; Lamb and Pugh, 2006).

IRBP binds retinoids (Adler and Spencer, 1991; Shaw and Noy, 2001) and is the most abundant soluble protein in the subretinal space (Loew and Gonzalez-Fernandez, 2002). In vitro studies have shown that IRBP promotes the release of all-trans retinol from photoreceptors (Ala-Laurila et al., 2006; Wu et al., 2007) and facilitates its delivery to the RPE (Okajima et al., 1994). Conversely, IRBP can promote 11-cis-retinal's release from the RPE (Edwards and Adler, 2000), prevent its isomerization in the subretinal space (Crouch et al., 1992), and transfer 11-cis-retinal to photoreceptors (Jones et al., 1989).

If IRBP were essential for any of these steps, its absence should inevitably cause 11-cis-retinal deficiency, and the Irbp ${ }^{-1-}$ mouse was expected to confirm IRBP's importance to the visual cycle in vivo (Liou et al., 1998). Although rod function is diminished in $\mathrm{Irbp}{ }^{-1-}$ mice, the visual cycle in rods is surprisingly normal (Palczewski et al., 1999; Ripps et al., 2000), and the reduced rod function is thought to result from degeneration (Liou et al., 
A

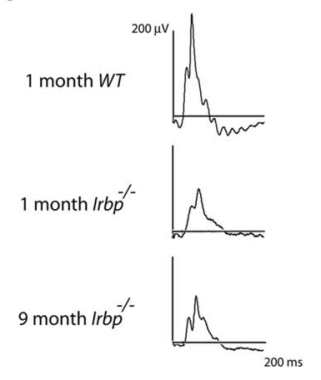

B

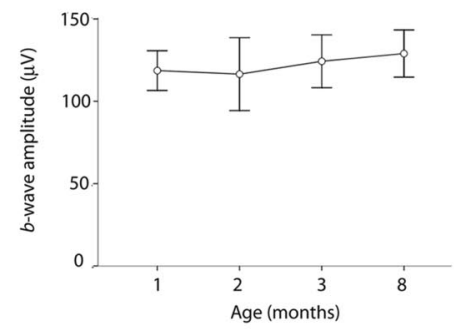

C

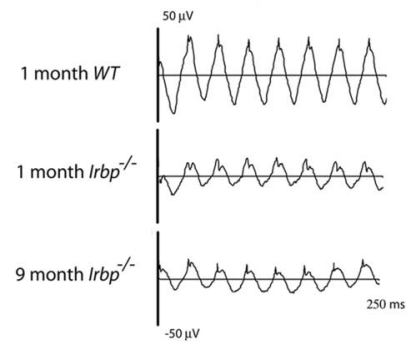

D

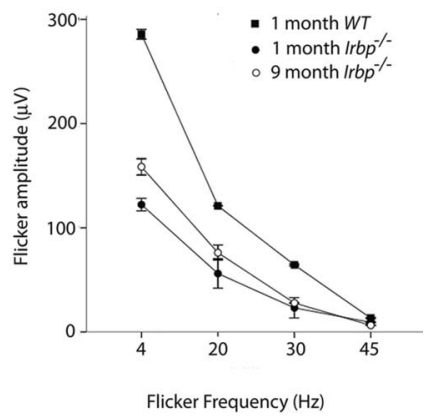

Figure 1. Cone function is stable in aging $/ r b p^{-1-}$ mice. $A$, Single-flash photopic ERG responses from individual WT and $/ r b p^{-1-}$ mice to a $0.4 \log \mathrm{cd}^{*} \mathrm{~s} / \mathrm{m}^{2} \mathrm{flash}$. B, Single-flash photopic ERGs $\left(0.4 \log \mathrm{cd}^{*} \mathrm{~s} / \mathrm{m}^{2}\right.$ stimulus) of $/ \mathrm{rbp}{ }^{-1-}$ mice at $1(n=9), 2(n=12), 3(n=13)$, and $8(n=12)$ months showed no significant change with age $(p=0.28,1$-way ANOVA). C, Thirty hertz Flicker ERG responses from individual WT and $/ \mathrm{rbp}{ }^{-1-}$ mice. $\boldsymbol{D}$, Flicker responses in $/ r b p^{-1-}$ mice $(n=3)$ were reduced relative to WT $(n=3)$, but remained stable from 1 to 9 months $(n=3)$. Data points represent mean amplitudes \pm SD.

1998). Cone function in $\operatorname{Irbp} p^{-1-}$ mice is also diminished (Ripps et al., 2000), but the underlying cause remains unclear. Our purpose was to identify the cause of cone dysfunction in $I r b p^{-1-}$ mice and determine whether a cone-specific visual cycle deficit exists.

\section{Materials and Methods}

Animals. Experimental procedures and animal care protocols adhered to the Association for Research in Vision and Ophthalmology Statement for the Use of Animals in Ophthalmic and Vision Research and were approved by the Institutional Animal Care and Use Committee of the Medical University of South Carolina. Control wild-type (WT) mice were adult C57BL/6J mice (The Jackson Laboratory). The Irbp ${ }^{-1-}$ mice were generated on a C57BL/6 background (Liou et al., 1998). Samples of each strain were genotyped to confirm the presence of the Leu450Met Rpe65 variant using the protocol described by Wenzel and coworkers (Wenzel et al., 2005).

Peanut agglutinin-stained retina flat-mounts. Eyes were dissected and placed in PBS ( $10 \mathrm{~mm}$ sodium phosphate, 0.15 m sodium chloride, $\mathrm{pH}$ 7.2). The retina-lens complex was fixed with $5 \%$ formaldehyde in PBS (4 $\mathrm{h}, 4^{\circ} \mathrm{C}$ ) and washed with PBS (three times, $30 \mathrm{~min}, 4^{\circ} \mathrm{C}$ ). 1:100 dilutions of FITC-conjugated peanut agglutinin (PNA) lectin $(0.2 \mathrm{mg} / \mathrm{ml}$; Sigma) were added $\left(12 \mathrm{~h}, 4^{\circ} \mathrm{C}\right)$. After washing with PBS (as above), the retina was separated and mounted on a slide. Anti-fade solution (ProLong; Invitrogen) was applied and images were acquired with fluorescent microscopy. Images were taken from the mid-dorsal and mid-ventral regions of the retina. Counts from both eyes were averaged to create a dorsal and ventral density for each animal. Dorsal and ventral densities were then averaged to yield an over-all cone density.

Western blots. Retinas were homogenized in 1\% SDS buffer, and equivalent amounts of total retina protein $(20 \mu \mathrm{g})$ were loaded onto $12 \%$ polyacrylamide gels following the XCell II Blot Module protocol (Invitrogen). Primary antibodies (1:500) for opsin detection were the rabbit anti-red/green opsin antibody (Millipore Bioscience Research Reagents) and rabbit anti-blue cone antibody (Millipore Bioscience Research Reagents). The secondary antibody (1:2000) was goat anti-rabbit IgG (Vector Laboratories). $\beta$-Actin was used as a loading control using mouse anti- $\beta$-actin (1:2000; Sigma) as a primary antibody and a goat antimouse IgG (1:5000; Vector Laboratories) secondary antibody. Blots were visualized with SuperSignal West Dura Extended Duration Substrate (Thermo Scientific), and images were captured on a VersaDoc Imaging System (Bio-Rad).

Retina cross sections. At room temperature, eyes were fixed in $4 \%$ paraformaldehyde ( $2 \mathrm{~h}$ ) and washed with PBS (three times, $15 \mathrm{~min}$ ). Eyecups were hemisected and cryoprotected in $30 \%$ sucrose (w/v) in PBS (overnight, $4^{\circ} \mathrm{C}$ ). Tissue was embedded in optimal cutting temperature (OCT) medium (Tissue Tek), sectioned through the dorsal-ventral plane $(14 \mu \mathrm{m})$, and washed with PBS. After blocking in a PBS-buffered solution (3\% BSA, 5\% normal goat serum, $5 \%$ donkey serum, and $0.4 \%$ Triton X-100) ( $1 \mathrm{~h}$, room temperature), the primary antibodies (1:200), rabbit anti-red/green opsin or rabbit anti-blue opsin cone (Millipore Bioscience Research Reagents), were applied to sections (overnight, $4^{\circ} \mathrm{C}$ ). Sections were then washed with PBS (as above) and incubated with donkey anti-rabbit Texas Red antibody (1:500; Jackson Immunoresearch) for $2 \mathrm{~h}$ at room temperature. Nuclei were stained with DRAQ5 (10 $\mu \mathrm{M}$; Biostatus Limited) for $10 \mathrm{~min}$. Images were acquired on a Leica confocal microscope. Sections used were made through the central retina (optic nerve visible). Images of the short-wavelength-sensitive (SWS) and medium-wavelength-sensitive (MWS) cone ospins were taken from the ventral and dorsal retina, respectively.

Electroretinogram analysis. Mice were anesthetized with xylazine (20 $\mathrm{mg} / \mathrm{kg}$ ) and ketamine $(80 \mathrm{mg} / \mathrm{kg})$, and pupils were dilated with phenylephrine hydrochloride $(2.5 \%)$ and atropine sulfate $(1 \%)$. Contact lens electrodes were placed on both eyes with a drop of methylcellulose. Electroretinogram (ERGs) were recorded with the UTAS E-2000 system (LKC Technologies) using $10 \mathrm{~ms}$ flashes of increasing light intensities under scotopic or photopic conditions. Cone recordings were made in the presence of a $30 \mathrm{~cd} / \mathrm{m}^{2}$ background light after $3 \mathrm{~min}$ of light adaptation.

Preparation and injection of 9-cis-retinal. 9-cis-retinal (Sigma) was prepared for injection under dim red light. The retinal was dissolved in ethanol ( $10 \%$ final injection volume) before adding the vehicle solution $(10 \% \mathrm{BSA}$ in $0.9 \% \mathrm{NaCl})$. After recording baseline ERGs, mice were rested $12 \mathrm{~h}$, administered $0.375 \mathrm{mg}$ of 9-cis-retinal intraperitoneally, and dark-adapted $12 \mathrm{~h}$ before recordings were repeated.

Statistical analysis. Statistical tests for each experiment were chosen after performing tests of normality for each sample (Kolmogorov-Smirnov test, Q-Q plots, and box plots). Photopic ERG responses of aging Irbp ${ }^{-1-}$ mice were analyzed by one-way ANOVA. Kruskal-Wallis and Mann-Whitney $U$ tests were used for cone density analysis. Intensity response functions between control and 9-cis-retinal injected Irbp ${ }^{-1-}$ mice were analyzed with a paired sample two-way ANOVA and a post hoc analysis using a paired samples $t$ test and the Bonferroni correction for multiple comparisons. Intensity-response functions from $\operatorname{Irbp}^{-1-}$ and WT mice were compared with a two-way ANOVA.

\section{Results}

Cone function in Irbp ${ }^{-/-}$mice with photopic ERGs

Cone responses in $\operatorname{Irbp}{ }^{-1-}$ mice are diminished at 1 month (Ripps et al., 2000) and would decline further if a degenerative process underlies cone dysfunction. Cone function was analyzed in aging $\mathrm{Irbp}^{-1-}$ mice with single-flash photopic and flicker ERGs. In agreement with previous findings (Ripps et al., 2000), cone responses were reduced in $I r b p^{-1-}$ mice at 1 month but were similar between $\operatorname{Irbp} p^{-1-}$ mice at 1 and 9 months of age (Fig. $1 A)$. Between 1 and 8 months of age, there was no difference in the cone responses of $\operatorname{Irbp} p^{-1-}$ mice to a $0.4 \log \mathrm{cd} \mathrm{s} / \mathrm{m}^{2}$ stimulus $(p=0.28)$ (Fig. $1 B$ ). Similar patterns of cone function were seen in flicker ERGs. Flicker ERG responses in $\operatorname{Irbp} p^{-1-}$ mice were 
A
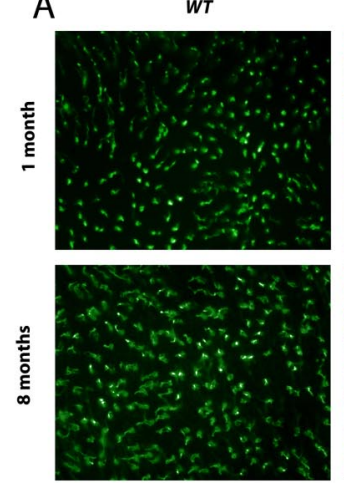

Irbp ${ }^{-1-}$

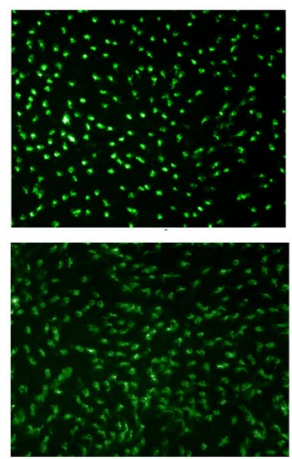

B

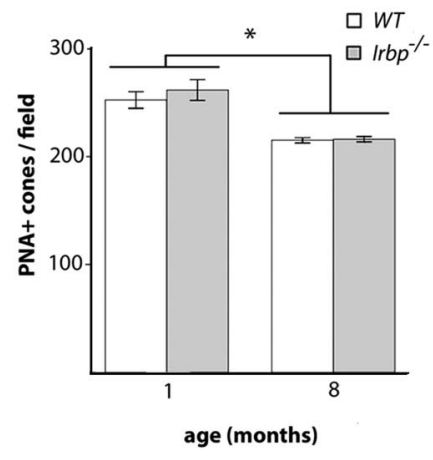

C

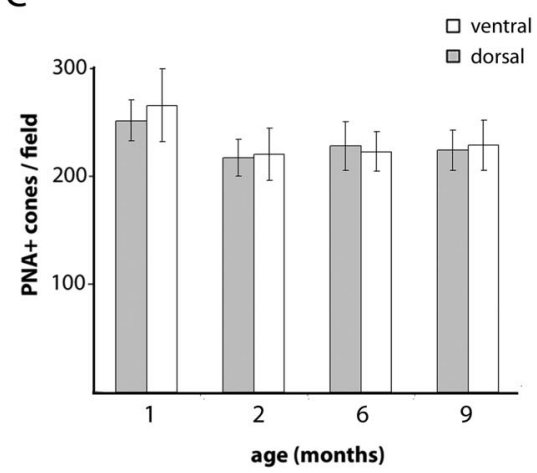

Figure 2. Cone densities in $/ r b p^{-/-}$and WT mice. $\boldsymbol{A}$, Images $(400 \times)$ of cones from PNA-stained (green) retina flat-mounts in WT and $/ r b p{ }^{-/-}$mice at 1 month and 8 months of age. $\boldsymbol{B}$, Cone densities were similar in Irbp ${ }^{-I-}$ and WT mice at 1 (Irbp ${ }^{-I-}, n=4 ;$ WT, $n=4 ; p=0.47$, Mann-Whitney test) and 8 (Irbp ${ }^{-1-}, n=3 ;$ WT, $n=3 ; p=1.00$, Mann-Whitney test) months. C, Cone densities counted from PNA-stained retina flat-mounts of $/ r b p^{-1-}$ mice at $1(n=4), 2(n=3), 6(n=4)$, and 9 ( $\left.n=4\right)$ months showed a drop between 1 and 2 months $(p=0.03$, Mann-Whitney test) but were stable between 2 and 9 months ( $p=0.14$, Kruskal-Wallis test). Densities were similar between the dorsal and ventral retina at all ages. All bars represent means \pm SD.

reduced relative to WT but similar between $\operatorname{Irb} p^{-1-}$ mice at one and 9 months (Fig. 1C,D). Scotopic ERGs showed reduced rod function in agreement with previous findings (Liou et al., 1998; Ripps et al., 2000). However, the constant level of cone function seen in both single flash and flicker ERGs from aging Irbp ${ }^{-1-}$ mice suggests that degeneration is not the primary cause of cone dysfunction.

Cone density in aging $\operatorname{Irb} p^{-/-}$mice While ERGs suggest that cone dysfunction is not secondary to degeneration, IRBP has proposed developmental functions (Gonzalez-Fernandez and Healy, 1990; Liou et al., 1994), and its absence could impair normal cone development. To determine if abnormalities in the cone population are present in Irbp ${ }^{-1-}$ mice, retina flat-mounts were stained with PNA, a lectin that binds the glycoprotein sheath surrounding cones (Lotan et al., 1975; Johnson et al., 1986), and cone densities were compared in Irbp ${ }^{-1-}$ and WT mice. For each retina, cone densities from the mid-dorsal and mid-ventral retina were used to generate an overall cone density. Figure $2 \mathrm{~A}$ shows representative flat-mounts from $\operatorname{Irbp} p^{-1-}$ and WT mice at 1 and 8 months. $\operatorname{Irb} p^{-1-}$ and WT mice had similar cone densities at 1 month $\left(\operatorname{Irbp}{ }^{-1-}, 256 \pm 4.3\right.$, $n=4$; WT, $261 \pm 11, n=4 ; p=0.47)$ and 8 months $\left(\operatorname{Irbp}{ }^{-/-}\right.$, $218 \pm 16, n=3$; WT, $216 \pm 13, n=3 ; p=1.0$ ) (Fig. $2 B$ ). Cone densities declined in both Irbp ${ }^{-1-}$ and WT mice showed a decline in cone density between 1 and 8 months. To determine whether specific cone subpopulations were affected in $\operatorname{rrb} p^{-1-}$ mice, densities were calculated for the dorsal and ventral retina of aging Irbp $p^{-1-}$ mice. In Irbp ${ }^{-1-}$ mice from 1 to 9 months, the overall cone densities dropped from 1 to 2 months $(256 \pm 4.3, n=4$; $222 \pm 5.3, n=4 ; p=0.03)$ but remained stable between 2 and 9 months $(p=0.14)$ (Fig. $2 C)$. At all ages, cone densities were similar in the dorsal and ventral retina, suggesting that neither the MWS or SWS cones are uniquely affected in $\operatorname{Irbp} p^{-1-}$ mice. In agreement with our ERG findings, cone densities were stable in aging $\operatorname{Irbp} p^{-1-}$ mice, and the similar cone densities between Irbp ${ }^{-1-}$ and WT mice suggest that abnormal development does not underlie cone dysfunction.

Cone opsin levels in Irbp $p^{-/-}$mice

Retina flat-mounts showed normal cone densities in $\operatorname{Irbp} p^{-1-}$ mice, but cone dysfunction could result from reduced or mislocalized opsin expression. Opsin levels and localization were analyzed with Western blots and immunohistochemistry. Western blots were performed for both cone opsins using equivalent levels of total retina protein and $\beta$-actin as a loading control. Representative blots from $\operatorname{Irbp}^{-1-}$ and WT mice at 4 months of age showed equivalent levels of MWS and SWS opsins (Fig. 3). Retina cross sections were also used to confirm the correct localization of cone opsins. Images showing the MWS and SWS opsins were taken from the mid-dorsal and mid-ventral retina, respectively, of Irbp ${ }^{-1-}$ and WT mice at 3 and 10 weeks. At both ages, the SWS and MWS cone opsins were localized to the outer segments of Irbp $p^{-1-}$ mice, and both cone populations were comparable to WT mice (Fig. 4). While loss of the outer nuclear layer evident between 3 and 10 weeks is consistent with the rod degeneration reported to occur in Irbp ${ }^{-1-}$ mice (Liou et al., 1998), the proper cone opsin localization and apparently healthy cone population suggest that that opsin abnormalities do not underlie cone dysfunction.

\section{Cone function in Irbp $p^{-1-}$ mice after exogenous 9-cis-retinal injections}

While neither the cone densities or opsin expression account for cone dysfunction in $\mathrm{rrbp}^{-1-}$ mice, reduced cone function could 


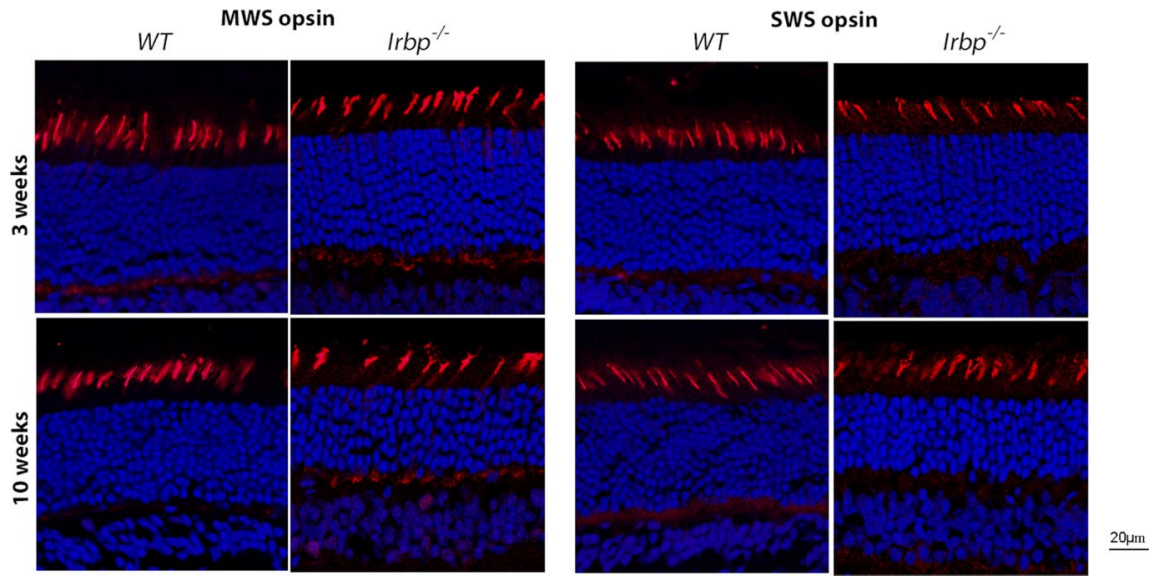

Figure 4. Cone opsin expression in $/ r b p^{-1-}$ and WT mice at 3 and 10 weeks of age. Confocal images of MWS and SWS opsins in $/ r b p^{-1-}$ and WT mice at 3 and 10 weeks show normal opsin localization. Sections $(630 \times)$ were stained with either anti-MWS or anti-SWS opsin antibodies (red). Nuclei were stained with DRAQ5 (blue). Images of MWS cone opsin were captured from the mid-dorsal retina, and images of SWS cone opsin were from the mid-ventral retina.

result from visual cycle deficits in IRBP's absence. We tested for 11-cis-retinal deficiency in the cones of $\operatorname{Irbp}{ }^{-/-}$mice by analyzing photopic ERGs before and after intraperitoneal injections of 9-cis-retinal, an 11-cis-retinal analog that forms visual pigments (Crouch and Katz, 1980). Baseline responses from $\operatorname{Irbp}^{-1-}$ mice were reduced relative to WT mice at all intensities but recovered dramatically after treatment with 9-cis-retinal (Fig. $5 A$ ). Intensity response plots from $\operatorname{Irbp}{ }^{-1-}$ mice $(n=8)$ show that cone responses increased significantly with 9-cis-retinal treatment at intensities above $-0.8 \log \mathrm{cd}^{*} \mathrm{~s} / \mathrm{m}^{2}(p=0.005)$ (Fig. $\left.5 B\right)$. While baseline responses from $\operatorname{Irbp} p^{-/-}$mice at each intensity were $50-$ $60 \%$ of the WT responses, treatment with 9-cis-retinal resulted in responses that were $80-100 \%$ of WT values (Fig. $5 C$ ). Furthermore, the responses of $I r b p^{-1-}$ mice treated with 9-cis-retinal did not differ significantly from the responses of treated WT mice $(p=0.25)$ (Fig. 5D). 9-cis-Retinal had no effect on rod function in $\operatorname{Irb} p^{-1-}$ mice ( $a$-wave, $p=0.70 ; b$-wave, $p=0.55$ ) (Fig. $5 E$ ), and did not significantly alter the rod or cone responses in WT mice. Thus, the cones of Irbp ${ }^{-1-}$ mice were uniquely sensitive to exogenous 9-cis-retinal, and the recovery of cone function to WT levels suggests that cone dysfunction in $\operatorname{Irbp}{ }^{-/-}$mice results from an 11-cis-retinal deficiency.

\section{Discussion}

\section{Rod and cone populations in Irbp ${ }^{-/-}$mice}

IRBP is thought to link the photoreceptors and RPE in the classical visual cycle. While its absence does not alter retinoid metabolism in rods (Palczewski et al., 1999; Ripps et al., 2000), our current findings show that IRBP is important to cone function. In vitro studies have shown that IRBP can facilitate photoreceptors by enhancing the removal of all-trans photoproducts (AlaLaurila et al., 2006; Wu et al., 2007) and the delivery of 11-cisretinal (Jones et al., 1989; Edwards and Adler, 2000). Yet, the flow of all-trans photoproducts between the rods and RPE is unimpeded in $I r b p^{-1-}$ mice, and even in the presence of the Leu450Met Rpe65 variant, 11-cis-retinal is regenerated at normal rates (Palczewski et al., 1999). Furthermore, normal rhodopsin regeneration rates in $\operatorname{Irbp} p^{-1-}$ mice suggest that the return of 11-cis-retinal to rods is normal (Ripps et al., 2000). The lack of response to 9-cis-retinal confirms that adequate 11-cis-retinal is available for rhodopsin regeneration (Fig. $5 E$ ) and provides additional evidence that the rod visual is normal in $\operatorname{Irbp}{ }^{-1-}$ mice.
As IRBP is not essential to the rod visual cycle, rod dysfunction in the model is likely the result of degeneration and could result from the accumulation of toxic photoproducts in the absence of IRBP's buffering abilities (Ho et al., 1989; Crouch et al., 1992; Wu et al., 2007). However, it is also likely that IRBP has important functions beyond retinoid transport. IRBP binds key outer segment components, such as docosahexanoic acid, that are essential for normal outer segment formation (Chen et al., 1993; Shaw and Noy, 2001). With disorganized outer segments common in the surviving rods of Irbp ${ }^{-1-}$ mice (Liou et al., 1998), shortages of essential outer segment components could underlie rod dysfunction. Additionally, its size, abundance, and light-dependent movement with the interphotoreceptor matrix (IPM) suggest that IRBP is an important structural component of the IPM (Uehara et al., 1990), and it is possible that the formation and support of rod outer segments is disrupted by an altered IPM in IRBP's absence.

While both rod and cone responses are reduced in $\operatorname{Irbp}{ }^{-1-}$ mice, our findings demonstrate a unique mechanism behind cone dysfunction. Stable cone ERGs (Fig. 1) and densities in aging Irbp ${ }^{-1-}$ mice (Fig. 2) suggest that a significant degenerative process does not mediate cone dysfunction, and similar cone densities and opsin levels to WT mice (Figs. 2-4) show that the cone population develops normally. Thus, cone dysfunction exists in the presence of surprisingly healthy cone population. Interestingly, an IRBP mutation has been identified in a subpopulation of retinitis pigmentosa patients, who showed a profound loss of rod function but retained residual cone function (den Hollander et al., 2008). Similarly, our findings show that $\operatorname{Irbp}{ }^{-1-}$ mice undergo rod degeneration but that cones are less profoundly affected.

\section{Recovery of cone function in Irb $p^{-/-}$mice with 9-cis-retinal} In the absence of significant degeneration or developmental abnormalities, reduced cone function in $\operatorname{Irbp} p^{-1-}$ mice is likely due to cone-specific visual cycle deficits. Rod and cone ERGs performed before and after 9-cis-retinal injections confirmed that visual cycle deficits in $I r b p^{-1-}$ mice are cone-specific (Fig. 5). Rod responses were unchanged by 9 -cis-retinal administration in $\operatorname{Irbp} p^{-1-}$ and WT mice, but the cone response of Irbp ${ }^{-1-}$ mice recovered to WT levels. While our findings do not differentiate between the two cone subpopulations, the use of a standard $\mathrm{Xe}$ non stimulator may have been more effective at eliciting responses from the MWS cones, which are similar to human L/MWS cones. However, rescue of the cone response with 9-cisretinal shows that at least the MWS cones survive with significant levels of unregenerated cone opsin and demonstrates that cones are uniquely dependent on IRBP for normal retinoid levels.

\section{Functions of IRBP in cone visual cycle}

Despite identifying a cone-specific visual cycle disruption, a number of questions remain surrounding IRBP's contribution to cone function. Two explanations are plausible based on our findings. First, in vitro studies suggest that IRBP contributes to the classical visual cycle by promoting 11-cis-retinal's release from 
A

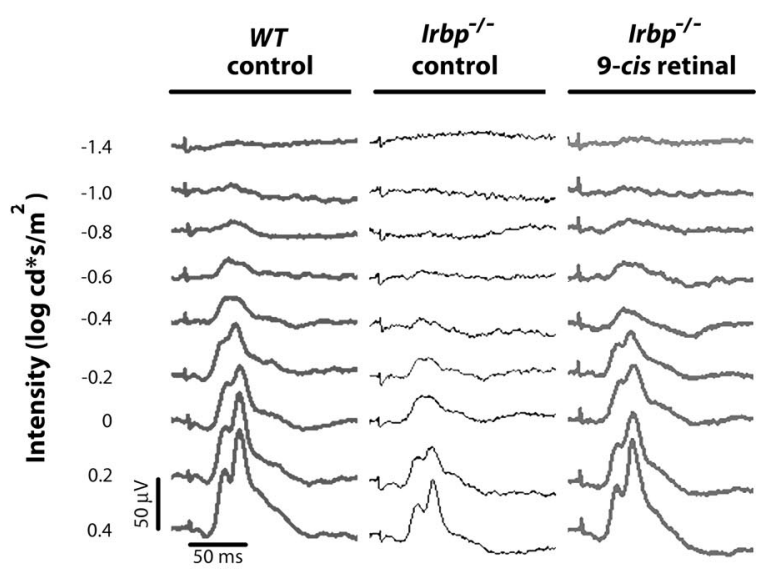

B

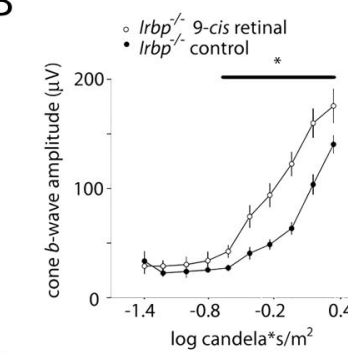

D

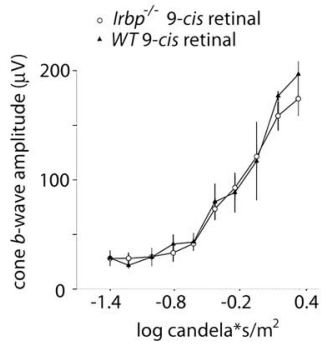

C

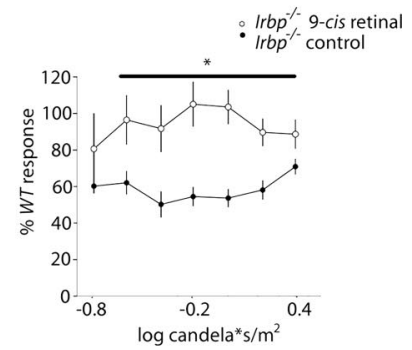

E

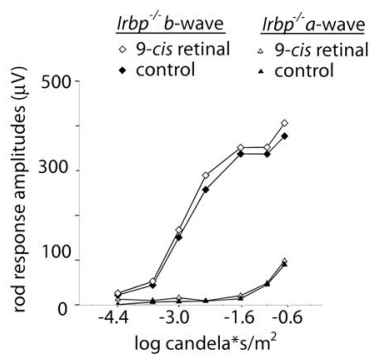

Figure 5. Recovery of cone ERGs in Irbp ${ }^{-1-}$ mice with exogenous 9-cis-retinal. $A$, Representative ERG traces from 2-month-old animals are shown. Control responses from the Irbp ${ }^{-1-}$ mouse were reduced relative to WT at all intensities. After the intraperitoneal injection of 9 -cis-retinal $(0.375 \mathrm{mg})$, responses from the same mouse recovered to WT levels. $B$, Intensity-response plots from ERG recordings of 2-month-old Irbp ${ }^{-1-}$ mice $(n=8)$ treated with 9-cis-retinal $\left(0.375 \mathrm{mg}\right.$, i.p.) showed a significant recovery of cone responses at all intensities above $-0.8 \mathrm{log} \mathrm{cd}^{*} \mathrm{~s} / \mathrm{m}^{2}(p=$ 0.005 , paired 2-way ANOVA). C, 9 -cis-Retinal injections resulted in cone responses between 80 and $100 \%$ of the WT response values. $\boldsymbol{D}$, Responses of WT $(n=4)$ and $/ \mathrm{rbp}{ }^{-/-}$mice after 9 -cis-retinal injections were not significantly different ( $p=0.25,2$-way ANOVA). $\boldsymbol{E}$, Scotopic ERGs of $/ \mathrm{rbp}{ }^{-1-}$ mice $(n=5)$ were no different after 9-cis-retinal injections. Error bars have been removed for clarity. All data points represent means \pm SD.

the RPE (Edwards and Adler, 2000) and delivery to photoreceptors (Jones et al., 1989). While the flow of 11-cis-retinal to rods is normal in $\mathrm{rrbp}^{-1-}$ mice (Ripps et al., 2000), the flow to cones has not been analyze, and it is possible that cones require IRBP to efficiently draw 11-cis-retinal from the RPE. A second explanation is that IRBP functions as the retinoid transporter in a proposed cone-specific visual cycle. Evidence in chicken and amphibian models (Hood and Hock, 1973; Jones et al., 1989; Das et al., 1992) suggests that cones have unique pathways for regenerating 11-cis-retinal through Müller cells (Das et al., 1992; Bustamante et al., 1995; Mata et al., 2002, 2005). Central to this theory is the unique ability of cones to regenerate 11-cis-retinal from 11-cis-retinol supplied to the inner segment (Jones et al., 1989). Müller cells can generate 11-cis-retinol (Das et al., 1992) and contain CRALBP in microvilli located near the cone inner segments (Bunt-Milam and Saari, 1983). IRBP colocalizes with the microvilli (Uehara et al., 1990), binds 11-cis-retinol endogenously (Saari et al., 1985), and is found in high concentration around cones (Carter-Dawson and Burroughs, 1992a,b). Although a functional relationship between IRBP, Müller cells, and cone inner segments has not been proven, the presence of retinoid deficient cones in $I r b p^{-1-}$ mice provides additional evidence for this pathway.

\section{Summary}

IRBP is not essential to the rod visual cycle, and the reduced rod function in $I r b p^{-1-}$ mice is likely from degenerative processes. Our current work shows that reduced cone function in $I r b p^{-1-}$ mice is due to 11-cis-retinal deficiency and implies that IRBP is essential to the normal cycling of retinoids in cones. These findings represent the first in vivo evidence implicating IRBP as a retinoid transporter in the visual cycle, and indicate a critical role for IRBP in the cone function that is essential for human vision.

\section{References}

Adler AJ, Spencer SA (1991) Effect of light on endogenous ligands carried by interphotoreceptor retinoid-binding protein. Exp Eye Res 53:337-346.
Ala-Laurila P, Kolesnikov AV, Crouch RK, Tsina E, Shukolyukov SA, Govardovskii VI, Koutalos Y, Wiggert B, Estevez ME, Cornwall MC (2006) Visual cycle: Dependence of retinol production and removal on photoproduct decay and cell morphology. J Gen Physiol 128:153-169.

Bunt-Milam AH, Saari JC (1983) Immunocytochemical localization of two retinoid-binding proteins in vertebrate retina. J Cell Biol 97:703-712.

Bustamante JJ, Ziari S, Ramirez RD, Tsin AT (1995) Retinyl ester hydrolase and the visual cycle in the chicken eye. Am J Physiol 269:R1346-R1350.

Carter-Dawson L, Burroughs M (1992a) Interphotoreceptor retinoidbinding protein in the cone matrix sheath. Electron microscopic immunocytochemical localization. Invest Ophthalmol Vis Sci 33:1584-1588.

Carter-Dawson L, Burroughs M (1992b) Interphotoreceptor retinoidbinding protein in the Golgi apparatus of monkey foveal cones. Electron microscopic immunocytochemical localization. Invest Ophthalmol Vis Sci 33:1589-1594.

Chen Y, Saari JC, Noy N (1993) Interactions of all-trans-retinol and longchain fatty acids with interphotoreceptor retinoid-binding protein. Biochemistry 32:11311-11318.

Crouch R, Katz S (1980) Effect of retinal Isomers on the VER and ERG of vitamin A-deprived rats. Vision Res 20:109-115.

Crouch RK, Hazard ES, Lind T, Wiggert B, Chader G, Corson DW (1992) Interphotoreceptor retinoid-binding protein and alpha-tocopherol preserve the isomeric and oxidation state of retinol. Photochem Photobiol 56:251-255.

Das SR, Bhardwaj N, Kjeldbye H, Gouras P (1992) Muller cells of chicken retina synthesize 11-cis-retinol. Biochem J 285:907-913.

den Hollander A, McGee TL, Ziviello C, Banfi S, Dryja TP, GonzalezFernandez F, Ghosh D, Berson E (2008) A homozygous missense mutation in the IRBP gene (RBP3) associated with autosomal recessive retinitis pigmentosa. Invest Ophthalmol Vis Sci. Advance online publication. Retrieved February 1, 2009. doi:10.1167/iovs.08-2497.

Edwards RB, Adler AJ (2000) IRBP enhances removal of 11-cisretinaldehyde from isolated RPE membranes. Exp Eye Res 70:235-245.

Fong SL, Liou GI, Landers RA, Alvarez RA, Bridges CD (1984) Purification and characterization of a retinol-binding glycoprotein synthesized and secreted by bovine neural retina. J Biol Chem 259:6534-6542.

Gonzalez-Fernandez F, Healy JI (1990) Early expression of the gene for interphotoreceptor retinol-binding protein during photoreceptor differentiation suggests a critical role for the interphotoreceptor matrix in retinal development. J Cell Biol 111:2775-2784.

Ho MT, Massey JB, Pownall HJ, Anderson RE, Hollyfield JG (1989) Mech- 
anism of vitamin A movement between rod outer segments, interphotoreceptor retinoid-binding protein, and liposomes. J Biol Chem 264:928-935.

Hood DC, Hock PA (1973) Recovery of cone receptor activity in the frog's isolated retina. Vision Res 13:1943-1951.

Johnson LV, Hageman GS, Blanks JC (1986) Interphotoreceptor matrix domains ensheath vertebrate cone photoreceptor cells. Invest Ophthalmol Vis Sci 27:129-135.

Jones GJ, Crouch RK, Wiggert B, Cornwall MC, Chader GJ (1989) Retinoid requirements for recovery of sensitivity after visual-pigment bleaching in isolated photoreceptors. Proc Natl Acad Sci U S A 86:9606-9610.

Lamb TD, Pugh EN Jr (2006) Phototransduction, dark adaptation, and rhodopsin regeneration the proctor lecture. Invest Ophthalmol Vis Sci 47:5137-5152.

Liou GI, Wang M, Matragoon S (1994) Precocious IRBP gene expression during mouse development. Invest Ophthalmol Vis Sci 35:1083-1088.

Liou GI, Fei Y, Peachey NS, Matragoon S, Wei S, Blaner WS, Wang Y, Liu C, Gottesman ME, Ripps H (1998) Early onset photoreceptor abnormalities induced by targeted disruption of the interphotoreceptor retinoidbinding protein gene. J Neurosci 18:4511-4520.

Loew A, Gonzalez-Fernandez F (2002) Crystal structure of the functional unit of interphotoreceptor retinoid binding protein. Structure 10:43-49.

Lotan R, Skutelsky E, Danon D, Sharon N (1975) The purification, composition, and specificity of the anti-T lectin from peanut (Arachis hypogaea). J Biol Chem 250:8518-8523.

Mata NL, Radu RA, Clemmons RC, Travis GH (2002) Isomerization and oxidation of vitamin A in cone-dominant retinas: a novel pathway for visual-pigment regeneration in daylight. Neuron 36:69-80.

Mata NL, Ruiz A, Radu RA, Bui TV, Travis GH (2005) Chicken retinas contain a retinoid isomerase activity that catalyzes the direct conversion of all-trans-retinol to 11-cis-retinol. Biochemistry 44:11715-11721.
Okajima TI, Wiggert B, Chader GJ, Pepperberg DR (1994) Retinoid processing in retinal pigment epithelium of toad (Bufo marinus). J Biol Chem 269:21983-21989.

Palczewski K, Van Hooser JP, Garwin GG, Chen J, Liou GI, Saari JC (1999) Kinetics of visual pigment regeneration in excised mouse eyes and in mice with a targeted disruption of the gene encoding interphotoreceptor retinoid-binding protein or arrestin. Biochemistry 38:12012-12019.

Pepperberg DR, Okajima TL, Wiggert B, Ripps H, Crouch RK, Chader GJ (1993) Interphotoreceptor retinoid-binding protein (IRBP). Molecular biology and physiological role in the visual cycle of rhodopsin. Mol Neurobiol 7:61-85.

Ripps H, Peachey NS, Xu X, Nozell SE, Smith SB, Liou GI (2000) The rhodopsin cycle is preserved in IRBP "knockout" mice despite abnormalities in retinal structure and function. Vis Neurosci 17:97-105.

Saari JC, Teller DC, Crabb JW, Bredberg L (1985) Properties of an interphotoreceptor retinoid-binding protein from bovine retina. J Biol Chem 260:195-201.

Shaw NS, Noy N (2001) Interphotoreceptor retinoid-binding protein contains three retinoid binding sites. Exp Eye Res 72:183-190.

Uehara F, Matthes MT, Yasumura D, LaVail MM (1990) Light-evoked changes in the interphotoreceptor matrix. Science 248:1633-1636.

Wald G (1935) Carotenoids and the visual cycle. J Gen Physiol 19:351-371.

Wald G (1955) The photoreceptor process in vision. Am J Ophthalmol 40:18-41.

Wenzel A, Grimm C, Samardzija M, Remé CE (2005) Molecular mechanisms of light-induced photoreceptor apoptosis and neuroprotection for retinal degeneration. Prog Retin Eye Res 24:275-306.

Wu Q, Blakeley LR, Cornwall MC, Crouch RK, Wiggert BN, Koutalos Y (2007) Interphotoreceptor retinoid-binding protein is the physiologically relevant carrier that removes retinol from rod photoreceptor outer segments. Biochemistry 46:8669-8679. 